\section{Overzicht van de bijdragen over 2013 (jaargang LXXII)}

\author{
[REDACTIONEEL] \\ Verdoodt (Frans-Jos) \\ De ongeschikte zesde provincie van Vlaanderen
} 299-302

De wereld is zozeer van de levenden en zo weinig van de doden ..3-6

Vérité au deçà de Pyrénées, erreur au delà......

99-102

Zweifeln ist menschlich

203-206

Toen de zon nog scheen over de vlasakkers van Vlaanderen

197-200

\section{[BIJDRAGEN \& BRONNEN]}

Absillis (Kevin), m.m.v. Lemmens (Wendy)

Het verdriet is gans het volk. Over Hugo Claus en de Vlaamse beweging 351-373

Boeva (Luc)

"Yet another book on nationalism". Enkele recente bijdragen tot de theorievorming .. 68-86 Dolderer (Winfried)

Een protestantse Flamenpolitik? 0tto Bölke - dominee, heemkundige, Jongvlaams propagandist 303-327

\section{Luyten (Dirk)}

Ondernemer tussen volk en staat. Tony Herbert: enkele historiografische ankerpunten vanuit biografisch perspectief 242-252 Monballyu (Jos)

Desertienaardevijand in het Belgischefrontleger tijdens de Eerste Wereldoorlog.

Deel 1 .. 39-67

Deel 2 140-175

Van den Bossche (Stefan)

De nalatenschap van een literaire duizendpoot. Over de heimatschrijver Omer Wattez (18571935) 328-350

Vandenbroucke (Dieter)

"De leer van de Daad". Het Vlaams activisme en het Duitse Aktivismus tussen nationalisme en kosmopolitisme 176-192

Vanlandschoot (Romain)

Verdraagzaamheid en pragmatische samenwerking in de Vlaamse beweging. Hugo Verriest en August Vermeylen 1895-1914.
Deel 1 7-38

Deel 2 103-139

Deel 3 207-241

\section{Verdoodt (Frans-Jos)}

Hector Plancquaert: daensistisch politicus voor, tijdens en na Daens, activist, geboren dissident 253-271

\section{[RECENSIES]}

Absillis (Kevin), Beeks (Sarah), Lembrechts (Kris) \& Wildemeersch (Georges) (red.)

De plicht van de dichter. Hugo Claus en de politiek, Antwerpen, De Bezige Bij, 2013 [Nico Van Campenhout] .374-376

Boehme (0livier)

De welvaart \& trots van naties. Een geschiedenis van het economische nationalisme, Antwerpen, De Bezige Bij, 2013 [Rik Van Cauwelaert] 193-194

De Boose (Johan)

Bloedgetuigen, Antwerpen, De Bezige Bij, 2011 [Bruno De Wever] 275-277

Monballyu (Jos)

Deserteurs voor de Vlaamse zaak. Over de Vlaamsgezinde militairen die naar de vijand overliepen, Brugge, De Klaproos, 2012 [Antoon Vrints] 195-197

Servaes (Paul)

Emile Verhaeren, Vlaams dichter voor Europa, Berchem, EP0, 2012 [Rik Hemmerijckx]

Turf (Jef)

Memoires. Van kernfysicus tot Vlaams communist, Tielt, Lannoo, 2012 [Harry Van Velthoven] 376-379

Van Cauwelaert (Rik)

"Ils nous ont pris la Flandre". Waals socialisme en Belgische illusies. Van Jules Destrée tot Elio Di Rupo, Kalmthout, Pelckmans, 2012 [Harry Van Velthoven] .................................. 277-281 Van Ginderachter (Maarten) \& Beyen (Marnix) (eds.)

Nationhood from below. Europe in the Long Nineteenth Century, Basingstoke, Palgrave Macmillan, 2012 [Lode Wils] ............. 281-283 Weijermars (Janneke)

Stiefbroeders. Zuid-Nederlandseletteren en natievorming onder Willem I, 1814-1834, Hilversum, Verloren, 2012 [Lode Wils] 272-275 


\section{[SIGNALEMENTEN]}

Couttenier (Piet) e.a. (red.)

Gezelliana. Kroniekvan de Gezellestudie, nummer 2011, Antwerpen, Universiteit Antwerpen, 2012 [Stefan Van den Bossche] .................. 91-92 De Franse Nederlanden

De Franse Nederlanden - Les Pays-Bas Français. 37ste jaarboek - 37es annales, Rekkem, Ons Erfdeel, 2012 [Frank Seberechts]............. 285 Goddeeris (Idesbald) \& Hermans (Roeland) (red.)

Vlaamse migranten in Wallonie (1850-2000), Leuven, KADOC-K.U.Leuven-Lannoo Campus, 2011 [Sophie Gyselinck] 286-287

Havenith (Guido)

Das Belgienbildim Grenz-Echo 1927-1940. Ein Weg der Integration?,Eupen, Förderverein des Archivwesensin der Deutschsprachigen Gemeinschaft Belgiens, 2012 [Frank Seberechts].. 288 Joris

Joris van Severen. Zijn persoon zijn gedachten zijn invloed zijn werk. Jaarboek 17, Ieper, Studiecentrum Joris van Severen, 2013 [Lode Wils] 383

\section{Lauwers (Herman)}

Links-liberalisme. Niemand is burger zonder land, Kalmthout, Pelckmans, 2012 [Harry Van Velthoven] .283-284

Nath (Giselle) \& Van Alstein (Maarten)

14-18 van dichtbij. Inspiratiegids voor lokale projecten over de Grote Oorlog, Leuven, Acco, 2012 [Sophie Gysenlinck] 289 Nuyttens (Michel)

Inventaris van het archiefvan en over Prudens Van Duyse (1804-1859) bewaard in de KANTL te Gent, Gent, Koninklijke Academie voor Nederlandse Taal- en Letterkunde, 2012 [Romain Van Eenoo].... 286

\section{Raskin (Brigitte)}

De taalgrens of wat de Belgen zowel verbindt als verdeelt, Leuven, Davidsfonds, 2012 [Harry Van Velthoven] .. 287

Rondas (Jean-Pierre)

De hulpelozen van de macht. Het federale graf van de Vlaamse regeringspartijen, Kalmthout, Pelckmans, 2012 [Sophie Gyselinck]......... 288 Schrans (Guy)

Van wit naar blauw. Gent tussen 1780 en 1842, Gent-Kortrijk, Snoeck-Liberaal Archief, 2010 [Harry Van Velthoven] 197
Toussaint (Jacques) (dir.)

Les Cahiers Wallons ont 75 ans. Les Rèlîs Namurwès auservice de l'identité wallonne, Namur, Province de Namur - Culture, 2012 [Frank Seberechts]

Van Campenhout (Nico) 287-288

Een man, zijn vrouw en hun twee zonen. Een collectieve biografie van de familie Pée, Gent, Academia Press, 2012 [Romain Van Eenoo] 284-285

Verstraete (Pieter Jan) (red.)

Cahiers Staf De Clercq. Bijdragen ter bevordering van de studie van Staf De Clercq en tijdgenoten, 6, Kortrijk, Verstraete, 2012 [Bruno De Wever]

Wauters (Karel)

HendrikConscience. Vangeboorte totleeuw, 18121838, Antwerpen-Brussel, Letterenhuis-ASP, 2012 [Romain Van Eenoo] .382-383 Zuurvrij

Zuurvrij. Berichten uithet Letterenhuis, december 2012, nr. 23 enjuni 2013, nr. 24 [Pietervan Hees] 379-382

\section{[REDACTIONELE MEDEDELINGEN]}

bij Wt, jg. 72, 2013, nr. 2, p. 164 [Jos Monballyu, Desertie naar de vijand in het Belgische frontleger tijdens de Eerste Wereldoorlog. Deel 2] ....... 291

\section{[ABSTRACTS]}

Absillis (Kevin)

The 'Sorrow (of Belgium)' applies to the entire nation. About Hugo Claus and the Flemish Movement. 385 Boeva (Luc)

"Yetanother book on nationalism". Some recent contributions to the generation of theories 94-95

Dolderer (Winfried)

A ProtestantFlamenpolitik(Flemish policy)? 0tto Bölke - protestant pastor, expert on local history, propagandist of the Young Flemish ... 384 Luyten (Dirk)

Entrepreneur between nation and state. Tony Herbert: some historiographical points of reference from a biographical perspective ...... 293 Monballyu (Jos)

Desertion to the enemy in the Belgian front line 
during the First World War.

Part 1

Part 2. 93-94 198-199

Van den Bossche (Stefan)

The legacy of a literary centipede. About the regional author Omer Wattez (1857-1935) 384-385

Vandenbroucke (Dieter)

"The doctrine of Action". Flemisch activism and German Aktivismus between nationalism and cosmopolitanism 199

Vanlandschoot (Romain)

Tolerance and pragmatic cooperation in the Flemish Movement. Hugo Verriest and August Vermeylen 1895-1914.

Part 1 .93

Part 2 198

Part 3 292

Verdoodt (Frans-Jos)

Hector Plancquaert: Daensist politician before, during and after Daens, activist, born dissident 293

[STUDIES ON NATIONAL MOVEMENTS] Introduction, articles and sources $386-388$

\section{[AUTEURS]}

Kevin Absillis

De Merodelei 4, 2600 Berchem

Luc Boeva

Tomveldstraat 22, 3000 Leuven

Bruno De Wever

De Berlaimontstraat 21, 2100 Deurne

Winfried Dolderer

Anna-Seghers-Strasse 96, 12489 Berlin

Sophie Gyselinck

Langhalsbeekstraat 120, 9100 Sint-Niklaas

Rik Hemmerijckx

Smidsestraat 109, 9000 Gent

Wendy Lemmens

Maandereind 10N, 6711 AC Ede

Dirk Luyten

Draaibankstraat 95, 2800 Mechelen

Jos Monballyu

Koning Bouwdewijnstraat 156, 8520 Kuurne

Frank Seberechts

Spoelstraat 9, 2580 Putte

Nico Van Campenhout

Zelestraat 6 bus 2, 9160 Lokeren

Rik Van Cauwelaert

Koning Albertstraat 115, 1760 Roosdaal

Stefan Van den Bossche

Resedalaan 2, 1770 Liedekerke

Dieter Vandenbroucke

Steenweg op Meerhout 18, 2430 Laakdal

Romain Van Eenoo

Asfilstraat 18, 9031 Afsnee

Pieter van Hees

Prof. Ritzema Boslaan 95, 3571 CP Utrecht

Romain Vanlandschoot

Hondstraat 4, 8700 Tielt

Harry Van Velthoven

Kloosterstraat 1, 1930 Zaventem

Frans-Jos Verdoodt

Massemsesteenweg 321.A, 9230 Massemen

Antoon Vrints

Simonslei 14, 2930 Brasschaat

Lode Wils

Graaf De Grunnelaaan 14, 3001 Heverlee 